\title{
Towards an Architecture of Collaborative Objects
}

\author{
Alexander Grasser ${ }^{l}$ \\ ${ }^{I}$ Institute of Architecture and Media, Graz University of Technology \\ Icontact@alexandergrasser.com
}

Towards an Architecture of Collaborative Objects, explores the potential of playing with Collaborative Objects in real, augmented and mixed realities. A multi-player game platform App: VoxelCO, developed by the author, provides a speculative playground to research, the interaction with objects, things and people, as well as provokes new opportunities to engage deeply with its content and context. Furthermore, VoxelCO, reveals new modes of participation, to design and collaborate in real-time with augmented reality, using millennial tools: mobile devices. A case study project, the VoxelStage, offered an opportunity to apply VoxelCO to design a stage together with a group of students. To merge the collaboratively aggregated virtual objects of VoxelCO with reality, real WireCubes were augmented and assembled, proposing an architecture of socially augmented fuzzy formations.

Keywords: Collaborative Objects, Augmented Reality, Realtime, Fuzzy, Play

\section{Introduction to Collaborative Objects}

"Technology has already transformed our lives, bringing together people, ideas, and information in unimaginable ways. We're hard at work in a new technological chapter that connects the digital world with reality"(Niantic 2019), a statement by Niantic, a company that recently blurred the boundary of what is digital or real on a massive scale. The expertise of $\mathrm{Ni}$ antic, developed among others from being involved in creating project Keyhole, which later evolved to Google Earth. Further on they developed their first geo-location based mobile game Ingress which allowed the users to claim virtual territories in the real world as well as enabled them to create content, documenting and mapping the real world and merging it with the virtual game. Building up on the collected data of Ingress, in 2016, Pokemon GO launched like a rocket, leading in the app charts for the follow- ing years. Besides the geo-location implemented in the game, advanced social concepts to collaborate, trade and raid, as well as augmented reality were introduced, mixing the realities of the game content. As Mario Carpo suggests, "Every technology is a social construction: innovation only occurs when technical supply matches cultural demand, and when a new technology and new social practices are congruent within the same techno-social feedback loop."(Carpo 2017, p.159)

I argue that social network platforms, enabling interaction with digital or real content, objects and people, as well as a technology like augmented reality allow for new forms of collaboration in architecture to emerge. It's time for architecture to investigate those 'unimaginable ways' to bring together people, ideas, information and objects. Therefore Collaborative Objects are introduced as conceptual 


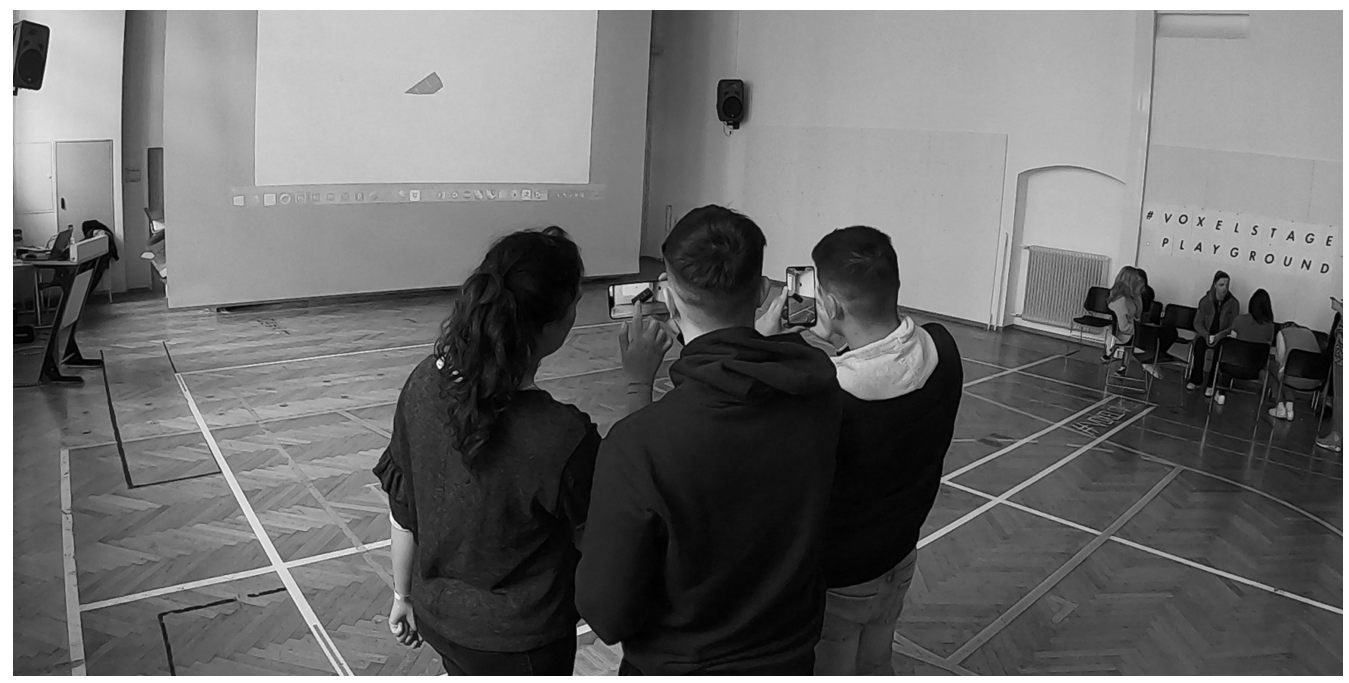

Figure 1

Millennial Tools to play VoxelCO at

Augmented

Playground

framework for the interaction between human to object, as well as object to object, in real or virtual environments and playgrounds towards an architecture of socially augmented fuzzy formations.

\section{Being Collaborative}

When Nicholas Negroponte portrayed our near future as, "The middle ground between work and play will be enlarged dramatically. The crisp line between love and duty will blur by virtue of a common denominator - being digital"(Negroponte 1996, p.220), we have to admit that we are not yet 'digital', but I would suggest we are close to 'Being Collaborative'. Enabled by the Internet and diffuse mobile devices, social network platforms and other forms of peer to peer production, together, empowered a culture of collaboration. As we recently learned again with Pokemon GO, a massively multi-player online game (MMO), "play is the central element through which cultures enact itself."(Bogost 2016, p76) MMO's enable players to connect to a main server, to get access to the game's digital playground. The server provides what's called a persistent game environment, a mechanism to ensure that the digital world map, all the changes a user makes to it, as well as the progress a player makes, is stored properly. This allows the digital playground to be a collaborative environment where users can interact with each other, with objects and their environment. Furthermore those open world games, with non linear play, enable in-game culture to emerge. Critically regarding contemporary production of architecture, there might be a shift towards social collaboration in the design process, as Jose Sanchez puts forward, from "growing form and producing differentiation by a omnipresent designer, the democratization of tools and the collaborative commons searches for massive social recombination, allowing differentiation not to be designed but to emerge from the interplay of resources and social innovation."(Sanchez 2014)

\section{Playing with Collaborative Objects}

Galit Ariel, offers some basic principles to explore our current reality:"We learn about our reality through exploration observation and play." (Ariel 2017, p.170) Exploration and observation are quite familiar in our reality of efficient architectural design and education, but usually there is not much serious or non-serious 
Figure 2

Virtual Voxel-

Formations at

Augmented

Playground, mobile

phone gameplay

screenrecordings

@VoxelCO

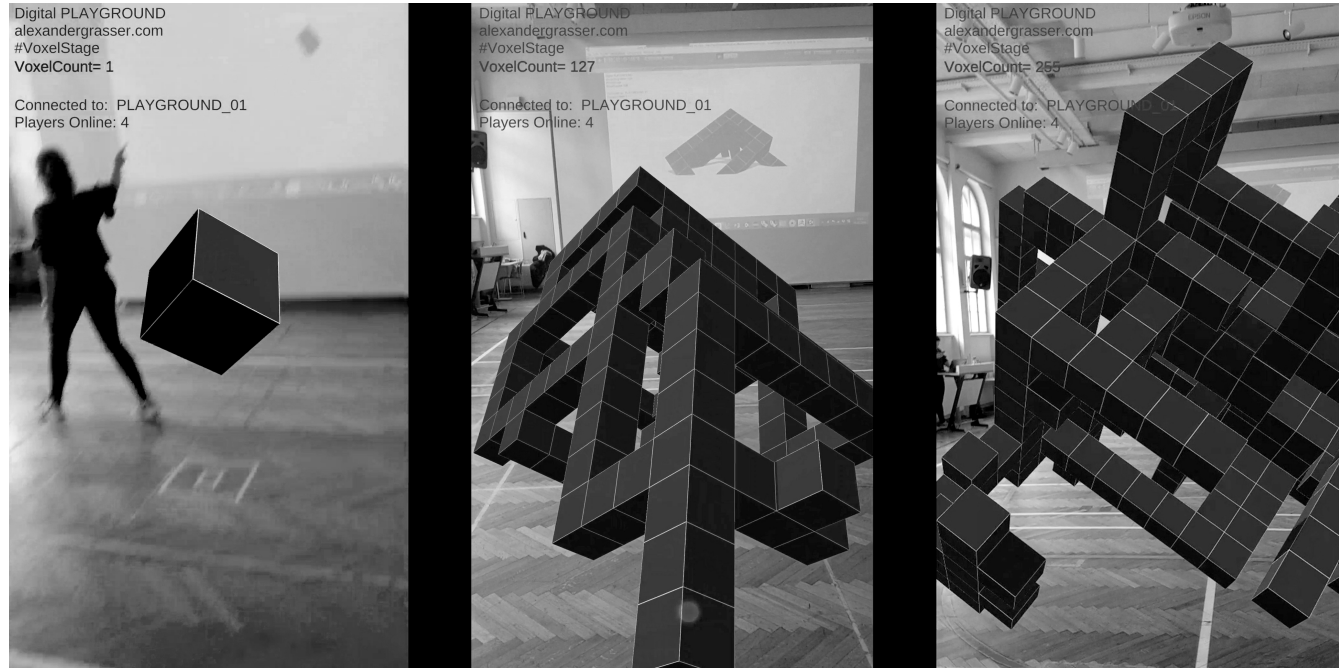

play involved. To collaborate and to play with clients, colleagues, students, objects and discrete units offers a huge potential as soon as we look at it, the way lan Bogost is looking at play:"Play is in things, not in you."(Bogost 2016, p.91) To play with Collaborative Objects in real, augmented and mixed realities we need to engage with them deeply until they reveal their capacities, potentials and something new. A familiar platform enabling interaction, collaboration and play: the playground, offers the potential to re-frame architecture in a speculative way. As lan Bogost mentions "it's content is real - but the frame of a playground helps make the real world partly fictional. It wraps an imagined boundary around something, allowing us to suspend temporarily our ordinary relationship with it as ground and to see it new as figure."(Bogost 2016, p.87)

By deliberately working and engaging with objects and things on a playground we might encounter some of their hidden capacities, as Bogost puts it: "Fun is the feeling of finding something new in a familiar situation."(Bogost 2016, p.6) Collaborative Objects are trying to find those moments of fun in a familiar architecture.

\section{VoxelCO}

To research the potential of engaging with Collaborative Objects, the App 'VoxelCO' was developed by the author. A collaborative multi-player platform, transreality game, cross-platform suitable for PC, IOS and Android. VoxelCO is based on a simple game logic of allowing players to place or remove virtual voxels to a persistent game environment. Furthermore VoxelCO allows for collaboratively playing and displaying the interaction with objects in real-time using augmented reality on mobile devices. As described by Galit Ariel: "As consequence of applying Augmented Reality our physical environment (and reality) would become a Sandbox platform - allowing users to freely manipulate and apply digital content within it."(Ariel 2017, p.125) , VoxelCo's social augmented reality provides a playground for intensive collaborative interaction with real and virtual content and players. As well as provides a tool to deeply engage with Collaborative Objects. The potential that Negroponte identified in the pixel, "The real power of the pixel comes from its molecular nature, in that a pixel can be part of anything,"(Negroponte 1996, p.125) shifts in VoxelCO from $2 \mathrm{D}$ to $3 \mathrm{D}$, from pixel to voxel. The voxel, a dis- 

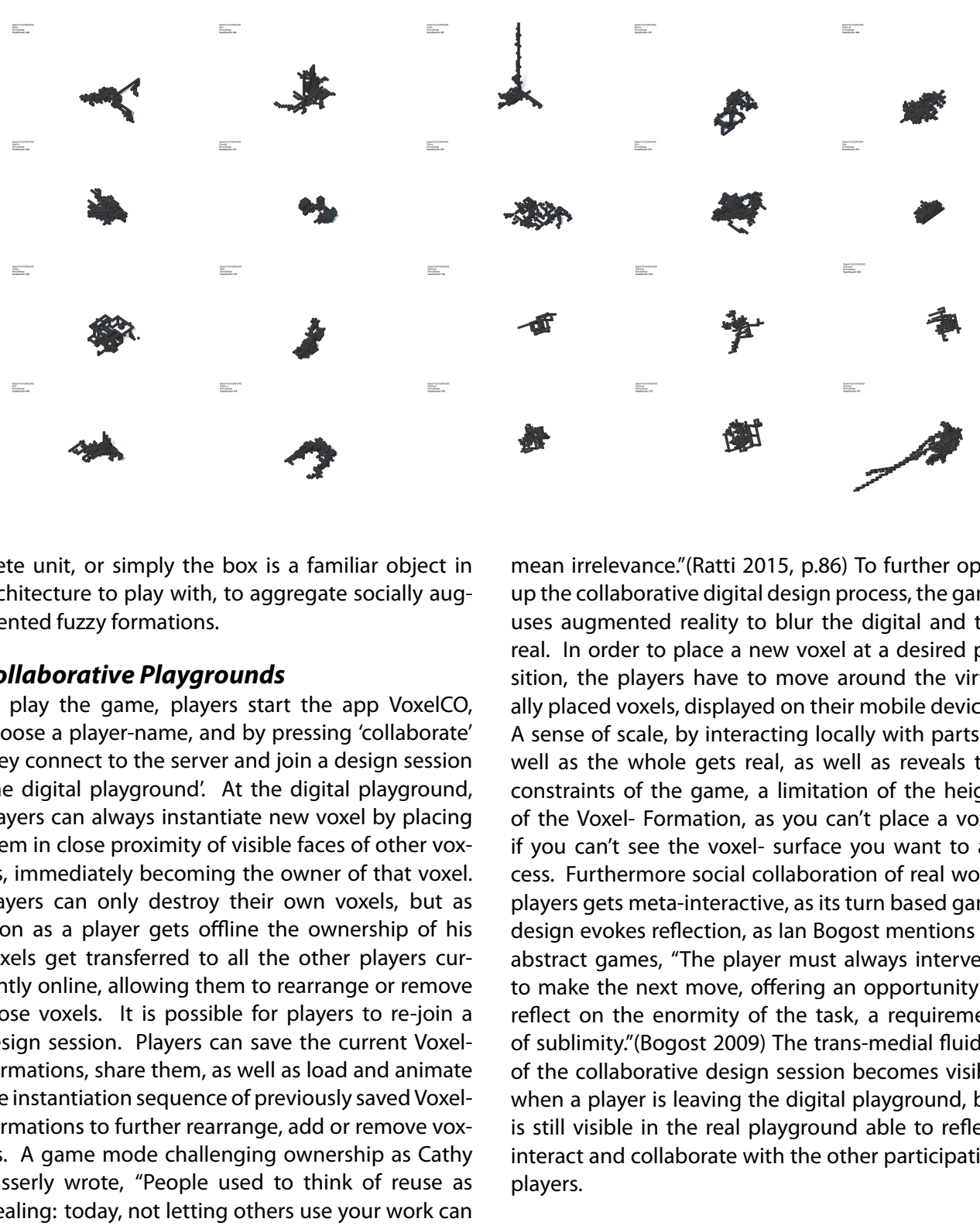

Figure 3

Virtual Voxel-

Formations at

Digital Playground

@VoxelCO

mean irrelevance."(Ratti 2015, p.86) To further open up the collaborative digital design process, the game uses augmented reality to blur the digital and the real. In order to place a new voxel at a desired position, the players have to move around the virtually placed voxels, displayed on their mobile devices. A sense of scale, by interacting locally with parts as well as the whole gets real, as well as reveals the constraints of the game, a limitation of the height of the Voxel- Formation, as you can't place a voxel if you can't see the voxel- surface you want to access. Furthermore social collaboration of real world players gets meta-interactive, as its turn based game design evokes reflection, as lan Bogost mentions on abstract games, "The player must always intervene to make the next move, offering an opportunity to reflect on the enormity of the task, a requirement of sublimity."(Bogost 2009) The trans-medial fluidity of the collaborative design session becomes visible when a player is leaving the digital playground, but is still visible in the real playground able to reflect, interact and collaborate with the other participating players. 
Figure 4

Virtual

Voxel-Formations +

Augmented

WireCube-

Formations at

Augmented

Playground

@VoxelCO

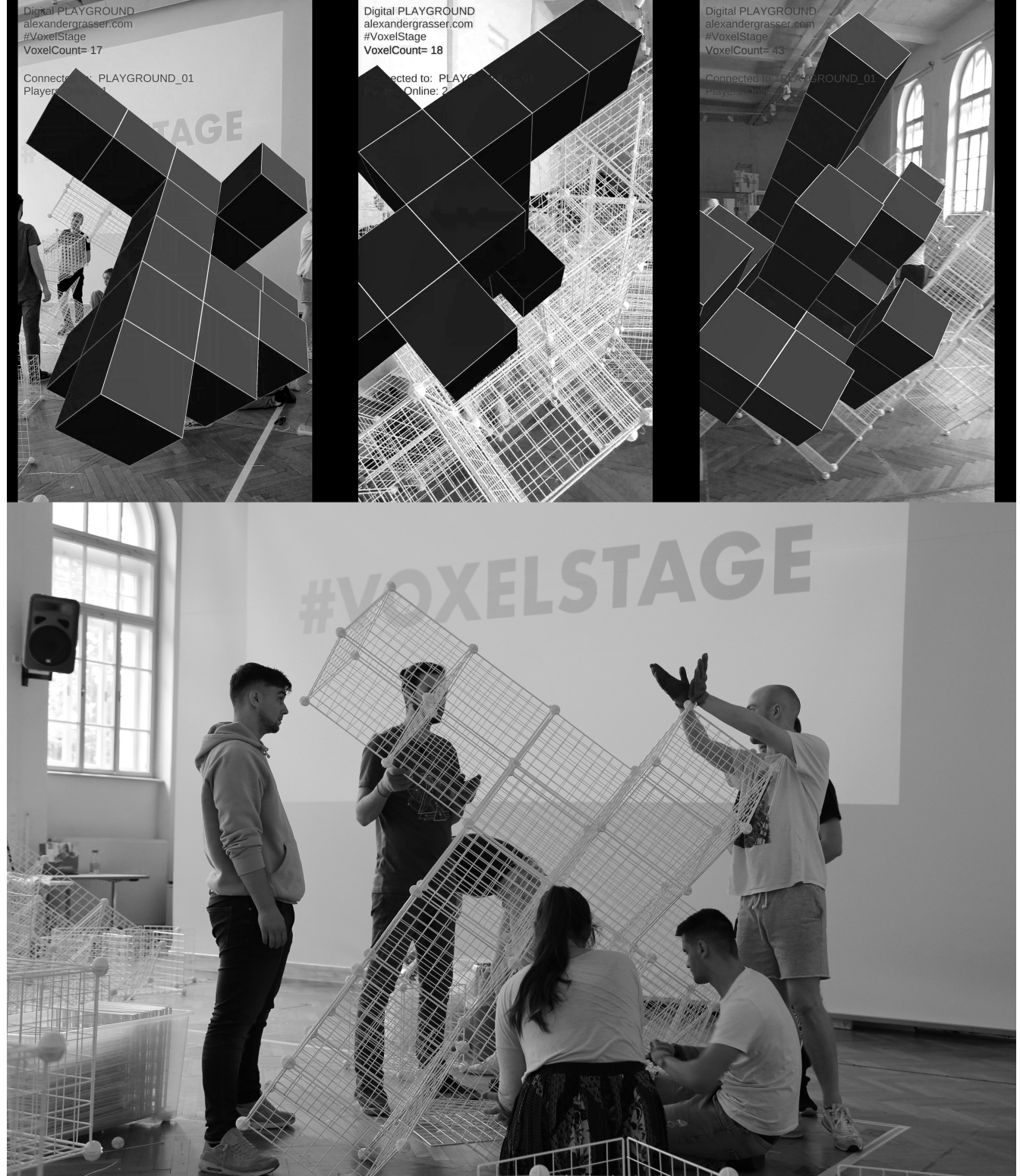

Design - COLLABORATION AND PARTICIPATION - Volume 1 - eCAADe 37 / SIGraDi 23 | 329 


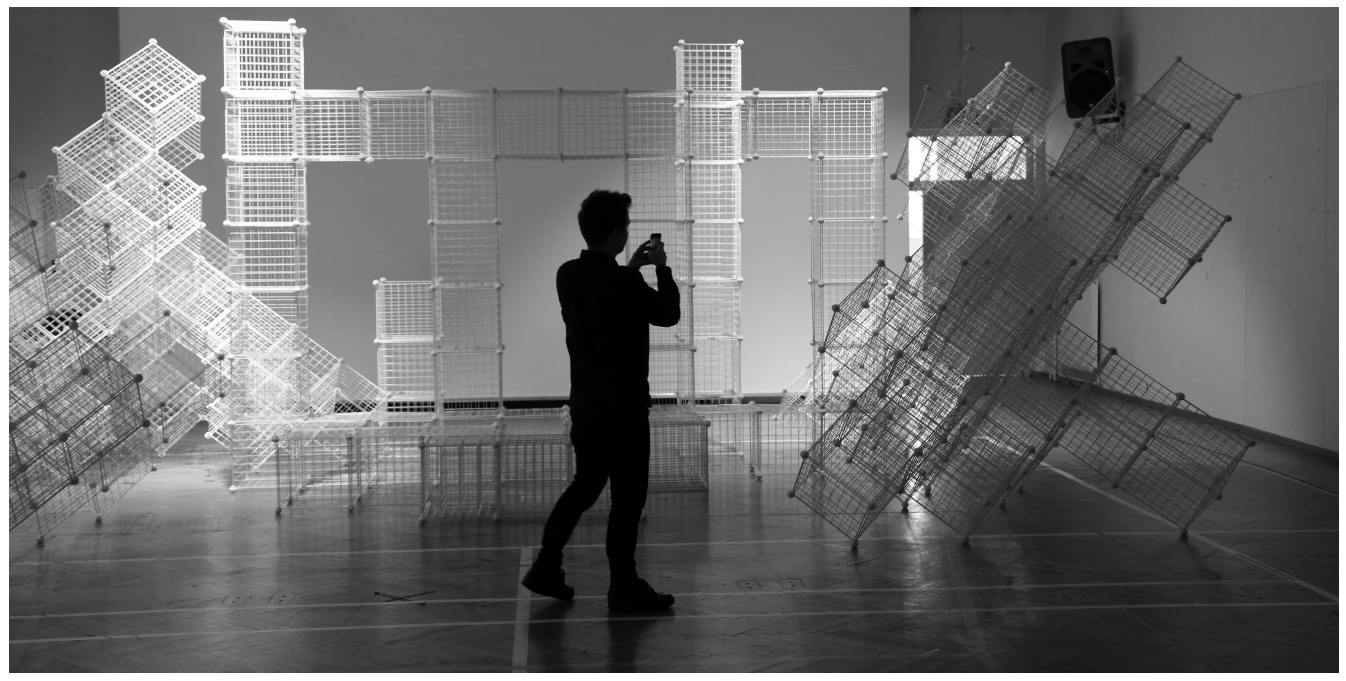

Figure 5

VoxelStage at

Augmented

Playground

\section{VoxelStage}

To apply the research of Collaborative Objects, a five day workshop with 20 bachelor students and the commission to design a modular stage for a festival offered a great opportunity to develop collaboratively a case study project, the 'VoxelStage'. The workshop explored the potential to merge the digital game of VoxelCO with our physical environment, as Carlo Ratti proposed "The kernel of architecture exists as data, is honed by a distributed sequence of adding and editing, and finally culminates in a physical structure - the execution of code in space."(Ratti 2015, p.109) The workshop was designed to teach the concept of developing a real-time multi-player platform with it's simple design logic, as well as to teach how to deploy the app on their mobile phones in an efficient way. Focusing on enabling the students to start playing with VoxelCO and to develop collaboratively virtual design proposals for the VoxelStage. Groups of 5 to 20 students designed at the real-time digital playground, as well as at the augmented playground, moving around to add or remove voxels, debating, arguing, designing and instantiating between 100 to 1000 voxels per design session. A Metaverse of Col- laborative Objects emerged. To execute the code in space, transferring the virtual VoxelStage to the real, students had to place real voxels, in our case WireCubes $(40 * 40 * 40 \mathrm{~cm})$ were provided. By using VoxelCO's function to load a instantiation sequence of a Voxel- Formation, loading one voxel after another, augmented reality provided a virtual overlay guiding mechanism to place the Wirecubes in our physical world, accelerating the assembly of stacking and connecting. The design sessions, alternated between playing with and aggregating virtual voxels in augmented reality, testing design options at the augmented playground, as well as analogue design sessions collectively aggregating the WireCubes to test their limits at the real playground. While playing and testing the platform VoxelCO to design the VoxelStage, adaptations and revisions of the application where made if needed. For example, disabling the ability to remove a voxel in design sessions was necessary, as it was sometimes not possible to remove a WireCube easily from a real WireCube- Formation without removing some neighboring WireCubes. Which as a side effect gave the instantiation of the virtual voxels more relevance and value. 
Figure 6

VoxelStage

\#VOXELSTAGE 
Furthermore, multiple design sessions with different constraints provoked different game strategies and modes of collaboration to emerge. From the fist access to the multi-player digital playground where the students came up immediately with 6000 instantiated virtual voxels, to design sessions with the goal of instantiating 400 virtual voxels, to design MetaVoxel-Formations of 20-40 virtual voxels as well as to build their related real WireCube- Formations.

VoxelCO framed a playground to mix and blend reality to deeply engage with Collaborative Objects. A collaborative design process similar to what lan Bogost describes "Most often, puzzles are entirely conceptual in form, with concreteness a mere accident of presentation."(Bogost 2009)

\section{Notes on the Metaverse of Collaborative Objects}

As the range of skills involved in setting up the game platform, deploying it to the phones, as well as to play the game, to connect the WireCubes and socially collaborate, with the goal of ending up with a sublime VoxelStage, I quote again Nicholas Negroponte describing a child being interviewed during a Lego Mindstorms Hackathon at MIT saying:"Yes, this is fun, but it's hard fun."(Negroponte 1996, p.196), along with a response to the introduction quote by Niantic I reply, as architect, "We're hard at work in a new technological chapter that connects the digital world with reality."(Niantic 2019)

\section{Acknowledgments}

VoxelCO Design, Development: Alexander Grasser VoxelStage Collaborators: Alexandra Parger (NanaDesign), Anna Brunner (Fifteen Seconds)

VoxelStage Tutors: Alexander Grasser, Alexandra Parger, Eszter Katona, Kristijan Ristoski

VoxelStage Students: Nadina Bajric, Emir Dostovic, Dijana Imsirovic, Jelena Josic, Larisa Kolasinac, Anela Mikic, Inas Dizarevic, Fabian Jäger, Matea Kelava, Bianka Marjanovic, Sali Ren, Sarah Salkovic, Anela Smajlovoic, Mirna Vujovic, Fabian Rigler, Cornelis Backenköhler

\section{REFERENCES}

Ariel, Galit 2017, Augmenting Alice: the future of identity, experience and reality, BIS Publishers

Bogost, lan 2009, 'Puzzling the Sublime', Accessed December 10. tinyurl.com/y6x8saot

Bogost, lan 2016, Play Anything: the pleasure of limits, the uses of boredom, and the secret of games, Basic Books

Carpo, Mario 2017, The Second Digital Turn: Design Beyond Intelligence, MIT Press

Negroponte, Nicholas 1996, Being Digital, Vintage Books

Niantic, Inc. 2019, 'The Niantic Story', Accessed January 20. www.nianticlabs.com/about

Ratti, Carlo 2015, Open Source Architecture, Thames \& Hudson Ltd

Sanchez, Jose 2014, 'Post-Capitalist Design: Design in the age of access', in Gerber, David Janson and Ibañez, Mariana (eds) 2014, Paradigms in Computing: Making, Machines, and Models for Design Agency in Architecture., eVolo Press 